physicians or other IBD-associated healthcare professionals, emergency room (ER) visits, hospitalizations and hospital admissions for surgeries); Health Related Quality of Life (HRQoL), pt-reported employment/UC-related sick leave status and Work and Productivity Activity Index (WPAI:GH; including work time missed (absenteeism), impairment while working (presenteeism), overall work productivity impairment, and daily activity impairment domains).

Results A total of 1816 UC pts were enrolled; mean \pm SD age was $38.5 \pm 14.6$ years and $833(45.9 \%)$ were female. At BL, 230 pts $(12.7 \%)$ were in remission, 672 pts $(37.0 \%)$ had mild UC, 668 pts $(36.8 \%)$ had moderate UC, and $234(12.9 \%)$ had severe UC. Compared to pts in remission, pts with moderate to severe UC had 1.8 to 2.6-fold higher rates of hospitalizations and 1.6 to 2.5 -fold higher rates of ER visits over the past 6 months. Pt with moderate and severe disease were associated with lower SIBDQ scores and higher WPAI:GH domain scores (i.e., greater impairment on work productivity) than pts with mild disease or those in remission.

$172(9.5 \%)$ pts reported to be unemployed at BL. 183 $(10.1 \%)$ reported sick leave at BL. Sick leave time ranged from $<2$ months (59.6\%), 2-4 months (12.6\%), >4 months (22.4\%).

Conclusion The direct and indirect burden of UC is substantial, as measured by healthcare resource utilisation and worklife impact. Pts with moderate and severe UC not only were associated with higher rates of urgent care in hospitalisation and ER visits, but also with poorer quality of life, higher unemployment, sick leave and impaired work productivity than pts with mild UC or in remission. For pts with mild UC, despite comparable HCRU to pts in remission, increased impact on work productivity remains a concern.

\section{PWE-068 ADVERSE EVENTS IN ELDERLY INFLAMMATORY BOWEL DISEASE PATIENTS MANAGED WITH ANTI-TNF THERAPY}

Conchubhair Winters*, Peter Mooney. Leeds Teaching Hospitals Trust, Leeds, UK

10.1136/gutjnl-2018-BSGAbstracts.200

Introduction In a population with an increasing life expectancy, a sizable proportion of inflammatory bowel disease (IBD) patients are elderly. The management of IBD often requires immunosuppressing anti-tumour necrosis factor (antiTNF) drugs which add to the immunosuppressive effects of ageing. Evidence for the safety of anti-TNF therapy in the elderly is scarce. Our objective was to assess the safety of antiTNF therapy in the elderly considering their co-morbidities and immunomodulators (IM).

\section{Methods}

Retrospective single centre study The IBD database of a large teaching hospital was interrogated for patients aged $>65$ years who had been prescribed infliximab or adalimumab. Patient electronic records were reviewed along with general practice prescribing records. Data was collected on co-morbidities, IM use, hospitalisations, significant adverse events (any reaction requiring discontinuation of the anti-TNF), and antibiotic prescriptions. Charlson Co-Morbidity index (CCI) was calculated. Results 80 patients (51 female) aged $>65$ received either infliximab $(n=50)$ or adalimumab $(n=30)$. Crohn's disease $(n=70)$ was more common and 34 patients were on a concomitant IM. The median duration of follow-up (FU) was 4 years and the median duration of therapy was 14 months. There were 5 deaths during FU, 4 after cessation of anti-TNF (2 pneumonias, 1 chronic obstructive pulmonary disease, 1 malignancy) and 1 patient was still on an anti-TNF (Crohn's related malnutrition). Seven patients developed cancer, 5 still on an anti-TNF and the other two were one and two years post-cessation of anti-TNF. Of the 5 patients who developed cancers on an anti-TNF, all 5 restarted their anti-TNF after treatment of the cancer. Eight patients (10.5\%) required hospitalisation due to what was felt to be an anti-TNF related event (7 infective, 1 allergic reaction). Patients on an IM had a $15.4 \%$ chance of anti-TNF related hospitalisation vs $4.4 \%$ in those not on a concomitant IM $(p=0.09)$. Concomitant IM use had no statistical impact on the risk of developing a cancer $(9.1 \%$ on an IM vs $6.5 \%$ not on an IM, p=0.49). Of those that required antibiotics, IM use did not seem to increase this risk $(p=0.43)$. Thirty one percent of those that stopped their anti-TNF $(n=50)$ did so because of an adverse event. When $\mathrm{CCI}=0$ was compared with a CCI $>0$, they were no more likely to still be on an anti-TNF after 12 months.

Conclusions In this series, we were unable to demonstrate a relationship between co-morbidities and tolerance of antiTNF therapy. There was, although not reaching statistical significance, a relationship between concomitant IMs and risk of hospitalisation due potential anti-TNF related events. Elderly patients are more likely to stop anti-TNFs than the younger populations used in larger trials. Concomitant IMs must be carefully considered to reduce the risk of adverse events.

\section{PWE-069 MEASURING THIOGUANINE NUCLEOTIDE (6-TGN) LEVELS AND CLINICAL RESPONSE IN IBD}

Mehmet Yalchin*, Lukasz Kamieniarz, Rigers Cama, Samuel Tribich, Andreas Koutsoumpas. Royal Free Hospital, London, UK

\subsection{6/gutjnl-2018-BSGAbstracts.201}

Introduction Monitoring levels of 6-TGN and titrating dose of Azathiopurine (AZA) and Mercaptopurine (6-MP) accordingly to achieve therapeutic concentrations of 6-TGN has been reported to improve outcomes in the treatment of Ulcerative colitis (UC) and Crohn's Disease (CD). The aim of our study was to show how levels of 6-TGN corresponds to clinical outcome.

Method This was a single centre (Royal Free Hospital), retrospective study, of patients receiving AZA or 6MP. We identified our patients by collating those who had been dispensed AZA or $6 \mathrm{MP}$ over the past 2 years. We were then able to access their electronic database, and record whether 6-TGN levels were subsequently taken to titrate treatment, and assess clinical outcome.

Results 109 from 426 patients have so far been analysed $(\mathrm{F}=54$ [50\%]). $\mathrm{UC}=45$ [41\%] $\mathrm{CD}=64$ [59\%].

Mean duration on thiopurines was 7.6 years. Mean disease duration was 16.2 years.

$74 / 109(68 \%)$ had 6-TGN levels at some stage.

42/74 (57\%) had therapeutic levels, whilst 32/74 (43\%) did not.

49/74 (66\%) were in clinical remission. 25/74 (34\%) were not. 